Revista

\title{
Multi-Ensayos
}

Vol. 7, núm. 13

ISSN: 2412-3285

https://multiensayos.unan.edu.ni

DOI: https://doi.org/10.5377/multiensayos.v7i13.10748

\section{Aprendizaje en las asignaturas "Electricidad" y “Termodinámica y Física Estadística" en tiempos de pandemia}

\section{Learning in the subjects "Electricity" and "Thermodynamics and Statistical Physics" in times of pandemic}

Cliffor Jerry Herrera Castrillo ${ }^{1}$

Recibido: 05 de octubre de 2020. Aceptado: 05 de noviembre de 2020

\section{RESUMEN}

El presente ensayo de relatos de experiencias, aborda la realidad que vive hoy la educación superior, siendo afectada por los efectos del COVID-19, es por ello que la manera de facilitar aprendizajes se ha tenido que rediseñar en todas las áreas, y la física como una de las ramas de la ciencia más importante en el campo del conocimiento humano, no se queda atrás. Es por ello que este ensayo fue realizado con el propósito de analizar experiencias vividas en IV y V año de la carrera de Física Matemática, durante el primer semestre 2020 en la UNAN - Managua, FAREM - Estelí, en las asignaturas de "electricidad" y "termodinámica y física estadística" en tiempos de pandemia, donde este proceso representó un gran reto tanto para los estudiantes como los maestros.

Palabras clave: aprendizaje; Física; Electricidad; Termodinámica; COVID-19.

\section{ABSTRACT}

The present essay of experiences, addresses the reality that higher education lives today, being affected by the effects of COVID-19, that is why the way of facilitating learning has had to be redesigned in all areas, and physics As one of the most important branches of science in the field of human knowledge, it is not far behind. That is why this essay was carried out with the purpose of analyzing experiences lived in the fourth and fifth year of the Mathematical Physics career, during the first semester 2020 at UNAN - Managua, FAREM - Estelí, in the subjects of "electricity" and "Thermodynamics and statistical physics" in times of pandemic, where this process represented a great challenge for both students and teachers.

Keywords: learning; Physics; Electricity; Thermodynamics; COVID-19.

1 Docente UNAN-Managua/FAREM-Estelí. Correo electrónico: clifforjerryherreracastrillo@gmail.com (c) 2021 Revista Multi-Ensayos. 


\section{INTRODUCCIÓN}

"La educación es el arma más poderosa que puedes usar para cambiar al mundo". Nelson Mandela

La pandemia de COVID-19 ha proyectado un desafío en todos los sistemas a nivel mundial, y la educación superior no se queda atrás, en donde ha sido necesario tomar medidas de "distanciamiento social" para aminorar la rapidez de contagio de la pandemia y así preservar muchas vidas humanas. El sector académico ha sido directamente afectado y forzado a modificar sus mecanismos pedagógicos para adecuarse al contexto actual y continuar facilitando aprendizajes con calidad y pertinencia, siempre con una visión humanística y científica. En este caso particular, es de interés analizar las experiencias en la carrera de física matemática en asignaturas específicas de física, durante el primer semestre 2020.

¿Cómo aprenden los estudiantes de física matemática, contenidos científicos de física en el contexto de pandemia de COVID-19?

La incógnita propuesta, envuelve, abordar a través del presente ensayo, como reflexionar sobre la práctica pedagógica, cómo facilitar aprendizajes, tomando en cuenta las realidades de los estudiantes, para poder sobrellevar sus estudios y estos puedan ser dotados de todos los conocimientos científicos y prácticos, que les serán de ayuda para su futuro desempeño laboral.

Para Sánchez, Payán, y Zeledón (2016) "la física permite desarrollar las capacidades de análisis, interpretación, síntesis a través de los conocimientos científicos, metodológicos e investigativos relacionados a la Ciencia Física que lo hacen capaz de desempeñarse en el ámbito laboral" (pp. 5)

Tomando en consideración lo planteado anteriormente, en este documento se presentan elementos que llevan a la reflexión de como el aprendizaje de la física es muy importante y a la vez de como la era digital, realmente ha llegado a la educación superior. Se deben de utilizar muchos recursos tecnológicos, que además de facilitar conocimientos, mediante la comunicación e información, ayuden a cumplir objetivos planteados en las asignaturas y seguir de forma permanente las medidas protocolarias para reducir los contagios de la pandemia mundial.

\section{DESARROLLO}

... "Nuestros estudiantes son el centro de nuestra presencia activa en la vida del país, y como tal, impulsaremos esfuerzos y estrategias para el desarrollo de los procesos de aprendizaje en los distintos turnos y modalidades. Los padres y madres de familia deben tener la confianza de que así lo haremos en beneficio de todas las familias y comunidades." Consejo Nacional de Universidades CNU, (2020).

La educación superior nicaragüense, ante la pandemia mundial toma la decisión de realizar clases de forma presencial, tomando en cuenta muchas medidas, para resguardar la salud de estudiantes, docentes y administrativos de las diferentes universidades del país. 
Basado en ese contexto, las carreras del turno sabatino de la FAREM ${ }^{1}$-Estelí, durante el primer semestre 2020 continuaron con las clases, bajo la modalidad de encuentros intercalados (es decir cada 15 días), donde se dividieron a los grupos con más de 30 educandos, se priorizaron contenidos que permitieran dar una base sólida en conocimientos a los estudiantes, y también la opción de trabajar aplicando muchos recursos tecnológicos, que facilitan los procesos de aprendizaje con calidad.

Una de las primeras preocupaciones que se identificaron es que la brecha digital hace más visibles las desigualdades. En América Latina y el caribe hay una baja conectividad a Internet, 45\%, y aunque las tasas de líneas móviles son elevadas (IESALC-UNESCO, 2020), el contrato de este servicio se hace principalmente a través de tarjetas de prepago o abonando saldo, por lo que algunos estudiantes, si sus padres se ven afectados por la crisis de desempleo, tendrán dificultad para tener acceso a Internet (UdeG, 2020).

La mayoría de estudiantes de las carreras de profesionalización², son de la zona rural, donde tiene poca conectividad, ahí es donde se vuelve importante la comunicación docente - estudiante, para establecer los mecánicos eficaces para todos, y así culminar con éxito, logrando los objetivos planteados.

Dentro de los medios de comunicación, que todos los estudiantes tienen acceso, están las redes sociales ya que de una u otra forma todos poseen, aunque sea una (Facebook, Messenger, WhatsApp, Instagram, entre otras), por lo que resultó pertinente trabajar mediante estos medios, además de que resultan más económicos para los estudiantes, un ejemplo de ello son las salas de Messenger, que consumen menos datos que utilizar Zoom, Meeting u otro recurso para video conferencias.

En el caso de las asignaturas de física "Electricidad" y "Termodinámica y Física Estadística", las cuales tienen un gran rigor científico, por ser clases propias de la carrera de física matemática, se trabajó con muchos medios de enseñanza, los cuales se detallarán a continuación.

\section{Medios audiovisuales}

Para el cumplimiento de las temáticas de Electricidad y Termodinámica y Física estadística, se realizaron muchos videos tanto de estudiantes como del maestro, con el fin de retroalimentar el proceso, realizar aclaraciones y explicar la resolución de problemas de forma cualitativa y cuantitativa, asimismo la realización de experimentos que permitieran comprender los fenómenos físico en estudio como: corriente eléctrica, campos eléctricos, intensidad voltaje, leyes de la termodinámica, entropía entre otros temas propios de las asignaturas analizadas.

Estos videos eran compartidos en los grupos de WhatsApp, donde se retroalimentaban con explicaciones, también en YouTube donde se podían reproducir las veces que fuese necesario, siendo los videos claros, precisos y concisos cumpliendo con el objetivo planteado en la clase y logrando aprendizajes en los estudiantes.

1 Facultad Regional Multidisciplinaria

2 Es un programa de Universidad Nacional Autónoma de Nicaragua, UNAN - Managua, donde se imparten clases por encuentros en cursos sabatinos, cuyo objetivo es la profesionalización de educadores empíricos procedentes de las diferentes regiones del país. 
Con el uso de este medio de enseñanza, se logró que muchos estudiantes vencieran el temor de hablar frente a cámaras, mejoraran su expresión oral, ya que en las clases habituales es solo el docente quien está explicando y el estudiante solo almacena esa información. Por medio de los videos se evidenció mayor participación del estudiante, utilizando el lenguaje científico de la clase y la interacción con materiales para realizar algún experimento, lo cual también mantuvo la motivación en toda la clase. Un ejemplo, es la siguiente figura:

\section{Figura 1}

\section{Videos de Electricidad}

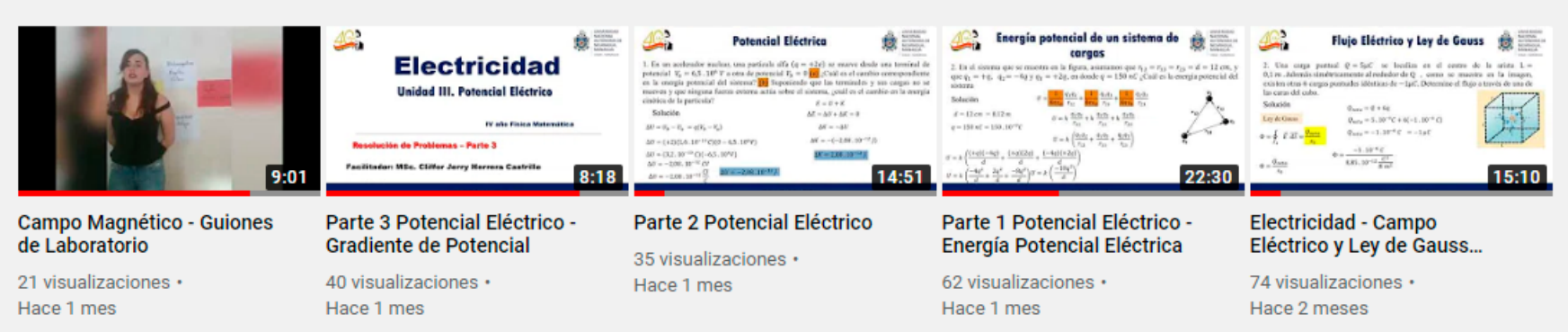

Fuente: YouTube [Link: https://www.youtube.com/channel/UCujXnrf-ibzvMGQe47SXBKQ/videos?view_ as $=$ subscriber]

En la asignatura de termodinámica y física estadística, cuando se trabajó la unidad III "Sistemas Termodinámicos Simples" en un encuentro presencial se explicaron los ciclos reversibles de Carnot ${ }^{3}$, Rankine ${ }^{4}$, Otto $^{5}$ y Diesel ${ }^{6}$, mediante exposiciones realizando experimentos, los cuales fueron grabados para así tener mayor interacción entre Grupo A y B (ya que para evitar aglomeraciones se dividieron los grupos de estudiantes en dos).

Con esa clase experimental, se logró ver la creatividad de los grupos al representar los diferentes ciclos reversibles, llevando la parte teórica a la práctica.

3 La máquina de Carnot es una máquina ideal que utiliza calor para realizar un trabajo. En ella hay un gas sobre el que se ejerce un proceso cíclico de expansión y compresión entre dos temperaturas.

4 El ciclo Rankine es un ciclo que opera con vapor, y es el que se utiliza en las centrales termoeléctricas. Consiste en calentar agua en una caldera hasta evaporarla y elevar la presión del vapor.

5 El ciclo Otto es característico de los motores de combustión interna, a gasolina, que encienden por la ignición de un combustible, provocada por una chispa eléctrica; se trata de un ciclo termodinámico en donde, teóricamente, el calor se aporta a un volumen constante.

6 El ciclo diesel es el ciclo ideal para motores de encendido por compresión. La bujía es sustituida por un inyector de combustible en los motores diesel. 


\section{Figura 2}

Exposición de los ciclos reversibles de Carnot, Rankine, Otto y Diesel

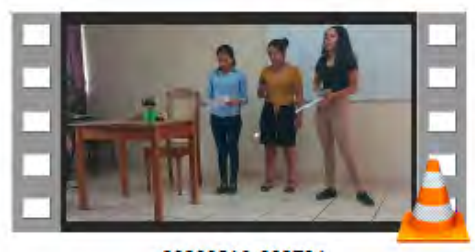

20200516_092704

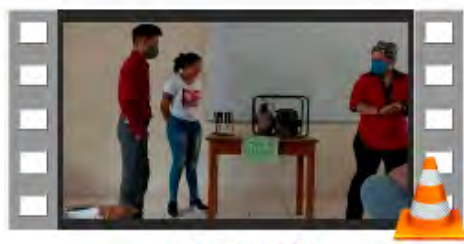

20200523_093122

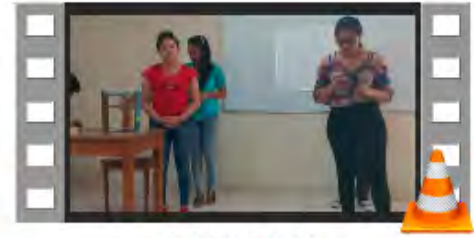

20200516_094015

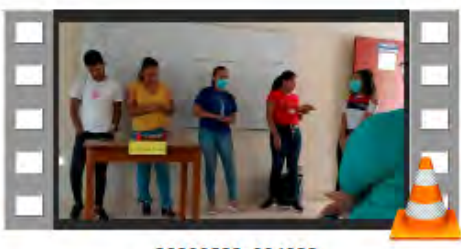

20200523_094822

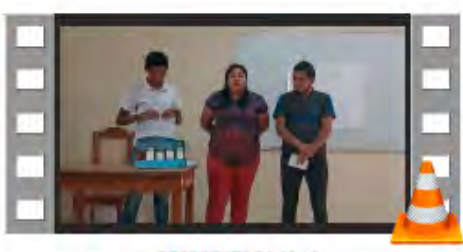

20200516_094841

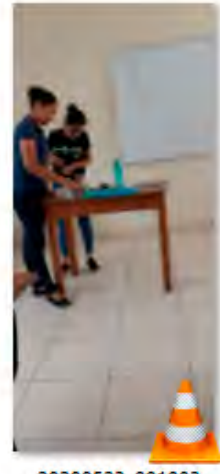

20200523091903

Fuente: El Autor

\section{Salas de Messenger}

Las salas de Messenger son una nueva forma de interactuar con personas en un chat de vídeo, esto permitió establecer clases virtuales, complementarias a las presenciales por encuentros con los estudiantes, donde se realizaban todas las aclaraciones necesarias y se abordaban temáticas nuevas. Para ser posible el uso de este medio, se acordó hora y día que la mayoría de estudiantes podía conectar e igual estas clases serian grabadas para ser subidas a YouTube, y quienes no se conectaran pudiesen verla después.

En la materia de termodinámica y física estadística se utilizó este medio, para lograr finalizar las unidades de Distribución de partículas en los sistemas y Estadística Cuántica, las cuales representan una base para la siguiente asignatura de "Física Cuántica", donde se aborda el mundo microscópico y los diferentes fenómenos que ocurren en él.

En el caso de electricidad, se utilizó la sala de Messenger, para la explicación de guiones de laboratorios, referente a temas vinculados a electricidad como campo eléctrico ${ }^{7}$, líneas de campo ${ }^{8}$, ley de $\mathrm{Ohm}^{9}$, capacitores ${ }^{10}$, corriente alterna y continua ${ }^{11}$, esto con el fin de consolidar los aprendizajes teóricos científicos con la práctica. Un ejemplo, es la siguiente figura:

\footnotetext{
7 Un campo eléctrico es un campo de fuerza creado por la atracción y repulsión de cargas eléctricas (la causa del flujo eléctrico) y se mide en Voltios por metro $(\mathrm{V} / \mathrm{m})$.

8 Son líneas imaginarias que ayudan a visualizar cómo va variando la dirección del campo eléctrico 9 La ley de Ohm se usa para determinar la relación entre tensión, corriente y resistencia en un circuito eléctrico. $10 \mathrm{El}$ capacitor es un dispositivo eléctrico que permite almacenar energía en forma de campo eléctrico 11 La Corriente Continua (CC) o Corriente Directa (DC) no varía su forma con respecto al tiempo, es decir, mantiene constante su valor máximo. La Corriente Alterna (AC) varía su valor entre un máximo y un mínimo de una forma senosoidal
} 


\section{Figura 3}

Clases mediante Salas de Messenger
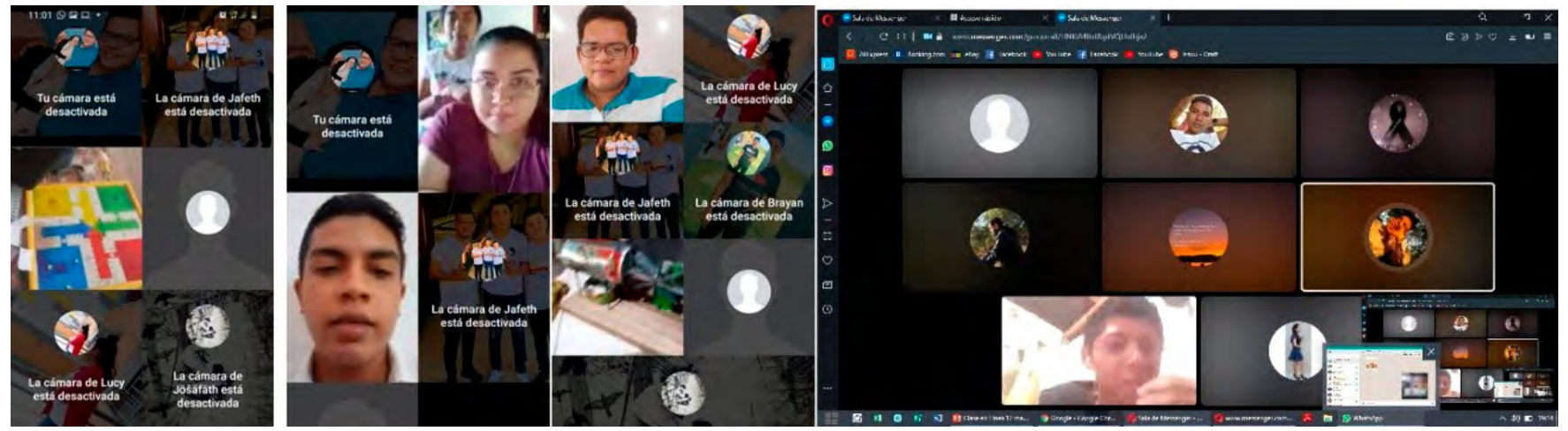

Fuente: El Autor

\section{WhatsApp y Classroom}

\section{Figura 4}

Información en Classroom

$11: 43 \pm 1$
$\leftarrow$

Información de la Unidad $\checkmark$ Conductividad Eléctrica

y Circuito de Corriente Eléctrica Continua

Saludos estimados, les comparto información referente a la Unidad 5 "Conductividad Eléctrica y Circuito de Corriente Eléctrica Continua"

Un gusto haber compartido esta asignatura con ustedes.

Corriente y resistencia, leyes de Ohmn Y Kirchhoff.pdf

Fuerza electromotriz para los circuitos eléctricos.pdf

$\mathrm{PDF}$ Funcionamiento de los instrumentos de medición eléctrica, tales

Agregar un comentario de la clase

III

Fuente: El Autor
Estos medios, ya se habían venido trabajando desde 2018, en la universidad para facilitar aprendizajes, con la pandemia COVID-19 se potencializó el uso de ellos para facilitar información y mantener la comunicación constante con los estudiantes.

Classroom es muy efectivo, para facilitar información de las asignaturas, asimismo para la entrega y revisión de trabajos asignados.

La entrega de trabajos gracias a Google Classroom puede realizarse de forma online. Así no es necesario imprimir los trabajos y hacer este gasto de papel. Esta aplicación facilita la entrega en formato digital de los trabajos de clase.

También, sirve poner al día a los estudiantes que no acuden a clase de forma presencial, Google Classroom es perfecto. El estudiante enfermo puede estar en contacto con las clases del profesor a través de Internet.

Por otro lado, mediante WhatsApp se realizaron debates interesantes sobre diferentes distribuciones de partículas microscópicas en la parte de termodinámica y física estadística, teniendo un acercamiento con el estudiante y este podía consultar sobre dudas o inquietudes, así como facilitar de forma veraz los contenidos. 
También esta herramienta, permite adjuntar grabaciones sonoras, vídeos, enlaces, fotos, imágenes y otros archivos. Formar grupos y trabajar con ellos y de esa forma facilitar aprendizajes. Como se muestra en la siguiente figura:

\section{Figura 5}

\section{Intercambio de experiencias mediante WhatsApp}

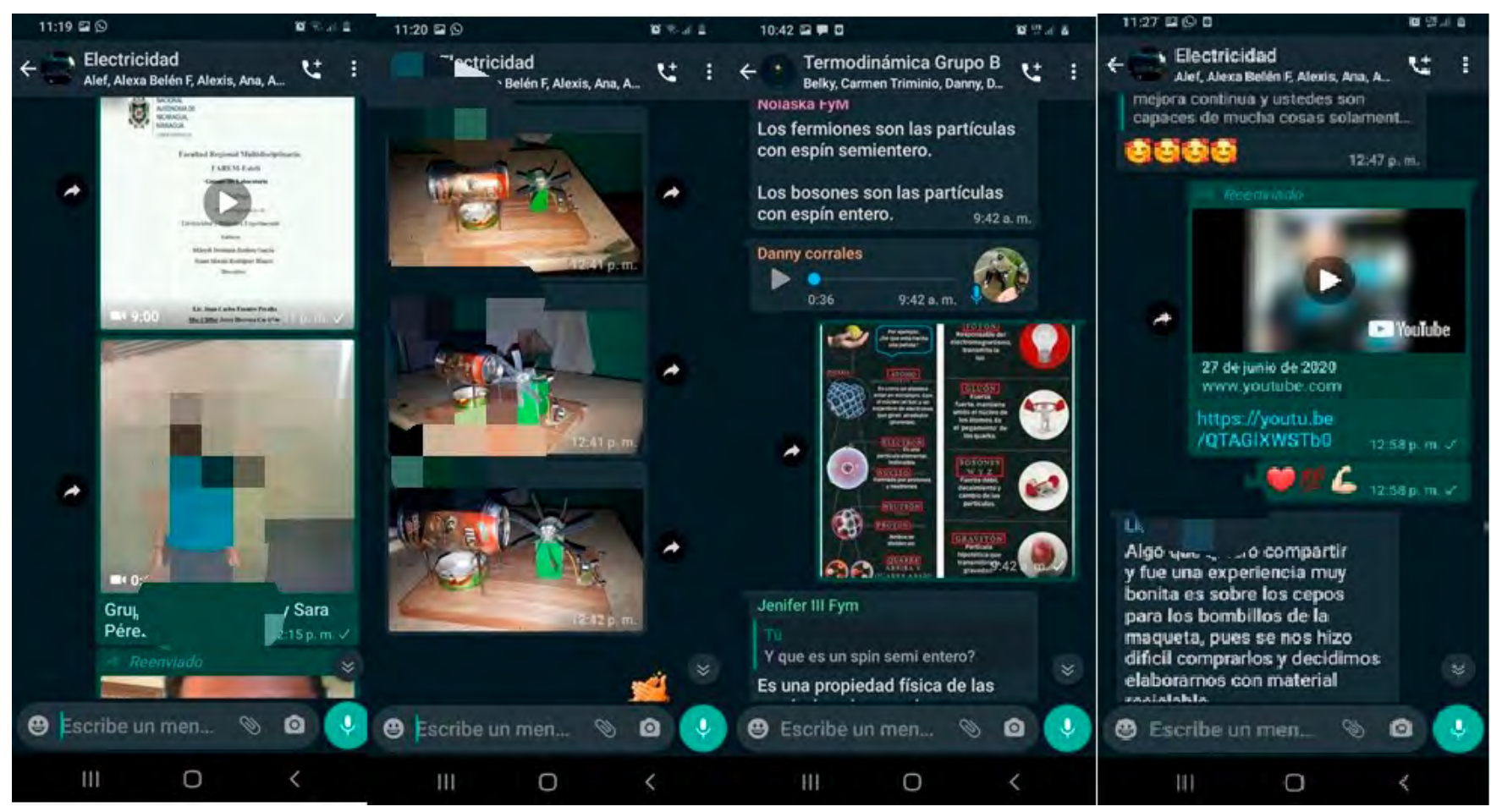

Fuente: El Autor

\section{Software Educativos}

Para el Ministerio de Educación (2008) "un software educativo es un valioso recurso utilizado en el campo de la educación, porque brinda la oportunidad de generar ambientes de aprendizajes interactivos y significativos". (pp.12)

El software educativo juega un papel importante en la educación, ya sea presencial, virtual o a distancia. Estas herramientas tecnológicas permiten que el estudiante se entusiasme más por aprender de clases científicas, como lo es la física, como por ejemplo los software OpenThermo ${ }^{12}$ y Proteus ${ }^{13}$.

Como se sabe, la física es una clase experimental, y al tener menos tiempo presencial con los estudiantes resulta necesario, recurrir a software educativos para realizar simulaciones de experimentos necesarios para la compresión y análisis de fenómenos físicos.

12 OpenThermo - Paquete de programas para cálculos de termodinámica estadística más allá del rotor rígido.

13 Proteus es una aplicación para la ejecución de proyectos de construcción de equipos electrónicos 
En la asignatura de Electricidad, se utilizó el programa Proteus, para explicar experimentalmente la ley de Ohm, reglas de Kirchhoff, corriente alterna y continua, entre otros contenidos.

\section{Figura 6}

Uso de Proteus

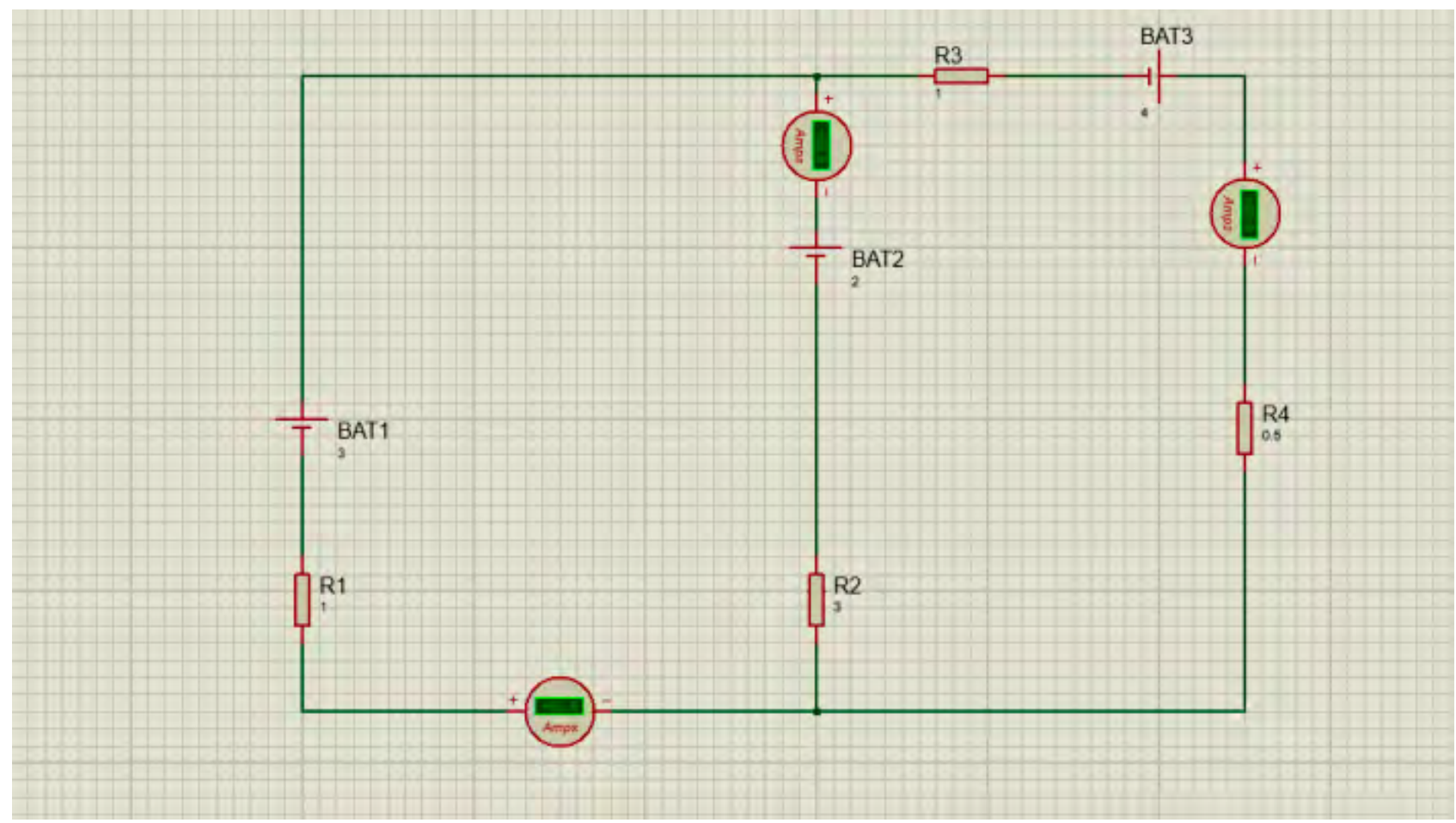

El Autor

Para realizar la simulación se parte de un circuito dado al inicio, como ejercicio donde se utilizarán elementos como lo son resistores y baterías también herramientas como lo es el amperímetro de corriente donde colocara 4 resistencias, también 3 baterías, donde se pueden cambiar los valores de acuerdo al ejercicio que se desee realizar, lógicamente para poder utilizar el programa se debe saber resolver el ejercicio manualmente.

A los estudiantes, les gusta el uso de este software, ya que les permite ampliar sus conocimientos, mediante la simulación de situaciones que ocurren a nivel microscópico y no son observadas a simple vista. También el uso de estos programas da la oportunidad de manejar recursos tecnológicos con mayor fluidez y así a futuro facilitar a próximas generaciones sus aprendizajes, debido a que todos los egresados de la carrera de física matemática se desempeñan en el ámbito educativo, principalmente la docencia. Para finalizar, no cabe duda que la educación superior debe adaptase a los cambios que se den por diferentes situaciones, en este caso la pandemia mundial COVID-19. Las Tecnologías de la información y la comunicación (TIC) juegan un papel muy importante, para el desarrollo de asignaturas de física, permite a los estudiantes comprender, analizar e interpretar de forma científica y práctica los contenidos y alcanzar los objetivos propuestos desde inicio del curso. 
La experiencia vivida en las asignaturas de física, fue diferente a la de los semestres anteriores, debido a que antes solo se hacía uso de medios para información y no se tenía la oportunidad de interactuar tanto con los estudiantes, como lo fue en el primer semestre 2020. Como docentes se deben asumir estos retos y continuar en actualización constante de los recursos que se pueden utilizar, para dar una educación con calidad y penitencia.

\section{CONCLUSIONES}

En este apartado se presenta una síntesis de las conclusiones a las que se llegó después de culminar los cursos de Electricidad y Termodinámica y Física estadística, con estudiantes de cuarto y quinto año de física matemática respectivamente, de la UNAN - Managua, FAREM - Estelí, durante el primer semestre 2020, acorde al cumplimiento de los fines planteados.

A continuación, se presentan las principales conclusiones de este escrito:

Con la pandemia COVID-19, los docentes tenemos el reto de adaptarnos al uso y manejo de la tecnología, para propiciar en los estudiantes un aprendizaje con calidad.

El uso de recursos tecnológicos, en asignaturas de física, despierta el interés de los estudiantes por las clase de física, además de que los dota de más habilidades y destrezas computacionales.

Las diversas acciones llevadas a cabo en la práctica pedagógica del desarrollo de las asignaturas de Electricidad y Termodinámica y Física estadística desde un entorno presencial y virtual ante el coronavirus, son de ayuda para mejorar capacidades y habilidades de los estudiantes, desarrollando sus competencias con mucha creatividad e innovación.

El uso de software educativos, para realizar simulaciones de fenómenos experimentales, permite en los estudiantes la mejor comprensión de los contenidos, ya que se vincula la teoría con la práctica de forma virtual.

El grupo de IV y V año de Física Matemática, con el que se trabajó consiguió realizar todas las tareas en línea, a pesar de esa brecha digital que existe en el país, los estudiantes lograron la manera de conectarse en los tiempos indicados, siempre manteniendo las medidas necesarias, frente a la pandemia.

\section{REFERENCIAS}

Consejo Nacional de Universidades CNU. (17 de Abril de 2020). COMUNICADO OFICIAL 02-2020 DEL CONSEJO NACIONAL DE UNIVERSIDADES. Comunicado de Prensa. Managua, Nicaragua.

IESALC-UNESCO. (6 de Abril de 2020). COVID-19 y educación superior: De los efectos inmediatos al día después. Análisis de impactos, respuestas políticas y recomendaciones. Obtenido de http://www. iesalc.unesco.org/wp-content/uploads/2020/04/COVID-19-060420-ES-2.pdf

Ministerio de Educación. (2008). Sugerencias para el uso pedagógico de las Tecnologías de la Información y Comunicación TIC. Managua, Nicaragua: PASEN. 
Sánchez Rizo, J. R., Payán, J. J., y Zeledón Meza, K. F. (2016). Programa de Asignatura Termodinámica y Física Estadística. Mangaua, Nicaragua: UNIVERSIDAD AUTÓNOMA DE NICARAGUA, MANAGUA FACULTAD DE EDUCACIÓN E IDIOMAS DEPARTAMENTO DE FÍSICA.

UdeG. (6 de Mayo de 2020). COVID-19 y educación desde casa. Obtenido de https://www.facebook.com/ udg.mx/videos/307119186936197/ 


\section{Anexos}

\section{Anexo A. Carta de autorización de uso de imagen (o datos personales) Autorizaciones}

24 noviembre del 2020

Estelí, Estelí

A:

Estudiantes de IV y V año de Física Matemática

Facultad Regional Multidisciplinaria de Estelí

Es un placer saludarles estimados, esperando que se encuentren bien todos, al lado de su familia.

El motivo de la presente es para hacerles, formal solicitud para utilizar su imagen y nombre en el ensayo "Aprendizaje en las asignaturas "Electricidad" y "Termodinámica y Física Estadística" en tiempos de pandemia", el cual será publicado en la segunda edición del año de la revista "Multi-Ensayos" de FAREM- Estelí, facultad de la Universidad Nacional Autónoma de Nicaragua, tiene como objetivo la publicación de ensayos inéditos que reflejan las experiencias metodológicas y didácticas de profesionales de la educación superior, al igual que el abordaje de experiencias que aporten en diversas áreas del conocimiento, con énfasis en temas vinculados al quehacer de la universidad y su incidencia en la comunidad universitaria.

Las imágenes mostradas en el ensayo son de videos durante la clases de "Electricidad" y Termodinámica y Física Estadística", también mensajes WhatsApp en el grupo de la clase donde se desarrolló una clase en línea, también capturas de salas de Messenger.

Esperando contar con su autorización, me despido fraternamente MSc. Cliffor Jerry Herrera Castrillo 


\section{Autorizaciones}

Samantha Lucia Cruz López

Keydin Ivania Ponce Morales
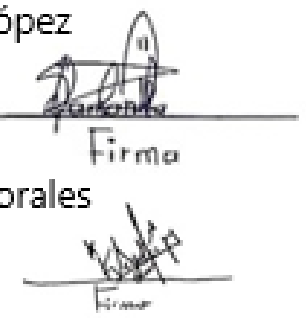

Dayana María Espinoza Rivas

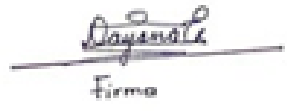

Lucy Yanixa López López

$$
\text { Firro: : }
$$

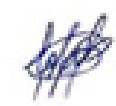

Sujeydi Yunieth Amador González

Danny A. Corrales Ochoa

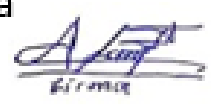

Deylin E. Ordoñez Moreno

$$
\text { Firme : क्रिमे }
$$

Aura Alina Martínez Benavidez
Jara Patricia Galeano Martínez

Keyling Y. Espinoza Palacios

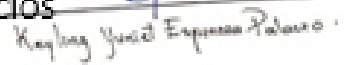

Mileydi Jerónima García Giménez

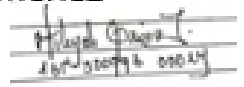

Jennifer del Carmen Ramírez Talavera

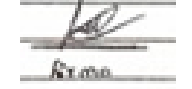

Meysi M. González Martínez

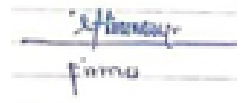
Jackeline N. Peralta Gutiérrez

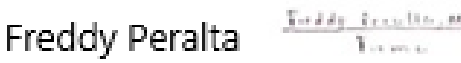

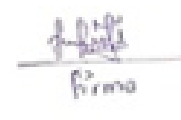

Eveling Amelia Inestroza López Li rma:

Kenia Maritza Suárez Seyilla

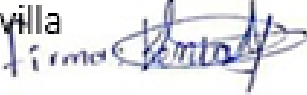

Eveling del Carmen Hernández

González

Osmary Elena Obregón Obregónd 